\title{
Statistical methods to derive efficacy estimates of anti-malarials for uncomplicated Plasmodium falciparum malaria: pitfalls and challenges
}

\author{
Prabin Dahal ${ }^{1,2^{*}}$, Julie A. Simpson ${ }^{3}$, Grant Dorsey ${ }^{4}$, Philippe J. Guérin ${ }^{1,2}$, Ric N. Price ${ }^{1,2,5}$ and Kasia Stepniewska ${ }^{1,2}$
}

\begin{abstract}
The Kaplan-Meier (K-M) method is currently the preferred approach to derive an efficacy estimate from anti-malarial trial data. In this approach event times are assumed to be continuous and estimates are generated on the assumption that there is only one cause of failure. In reality, failures are captured at pre-scheduled time points and patients can fail treatment due to a variety of causes other than the primary endpoint, commonly termed competing risk events. Ignoring these underlying assumptions can potentially distort the derived efficacy estimates and result in misleading conclusions. This review details the evolution of statistical methods used to derive anti-malarial efficacy for uncomplicated Plasmodium falciparum malaria and assesses the limitations of the current practices. Alternative approaches are explored and their implementation is discussed using example data from a large multi-site study.
\end{abstract}

Keywords: Plasmodium falciparum, Kaplan-Meier, Cumulative incidence function, Competing risks, Comparative studies

\section{Background}

Despite a rich pharmacopeia of anti-malarial agents, the emergence and spread of anti-malarial drug resistance has been relentless, with resistance now documented to all recommended treatment regimens in widespread use. Efficacy estimates derived from in vivo clinical trials, including post marketing and surveillance studies, form the basis for monitoring the status of anti-malarial drug resistance, with in vitro drug susceptibility testing and molecular analyses providing important complementary and confirmatory information. The main measure of anti-malarial clinical efficacy is the risk of recrudescent infection, which is defined as recurrent parasitaemia with parasites that were present prior to the initiation of treatment. Recrudescence needs to be differentiated from

\footnotetext{
*Correspondence: prabin.dahal@wwarn.org

${ }^{2}$ Centre for Tropical Medicine and Global Health, Nuffield Department of Clinical Medicine, University of Oxford, Old Road Campus, Roosevelt Drive, Oxford OX3 7FZ, UK

Full list of author information is available at the end of the article
}

reinfections arising from inoculation with a new parasite strain during the follow-up period. New infections can be either from the same parasite species or a different one (Fig. 1) [1].

Recrudescent parasitaemia arises from a variety of factors which can be broadly categorized into those related to the host, the parasite or the drug (Fig. 2). Pharmacokinetic factors can be ameliorated by improved prescribing practices, such as revising the dosing strategy and co-administration with food, whereas significant drug resistance usually requires revision to an alternative treatment regimen. At patient level, failure to achieve adequate clinical cure (i.e. preventing recrudescent infections) can have serious clinical implications including cumulative risk of anaemia, rising parasitaemia, and early treatment failure, which individually and collectively may lead to severe malaria and even death [1]. At the community level, treatment failures can lead to an increased economic burden and onwards transmission, fuelling the development of anti-malarial drug resistance [2]. 


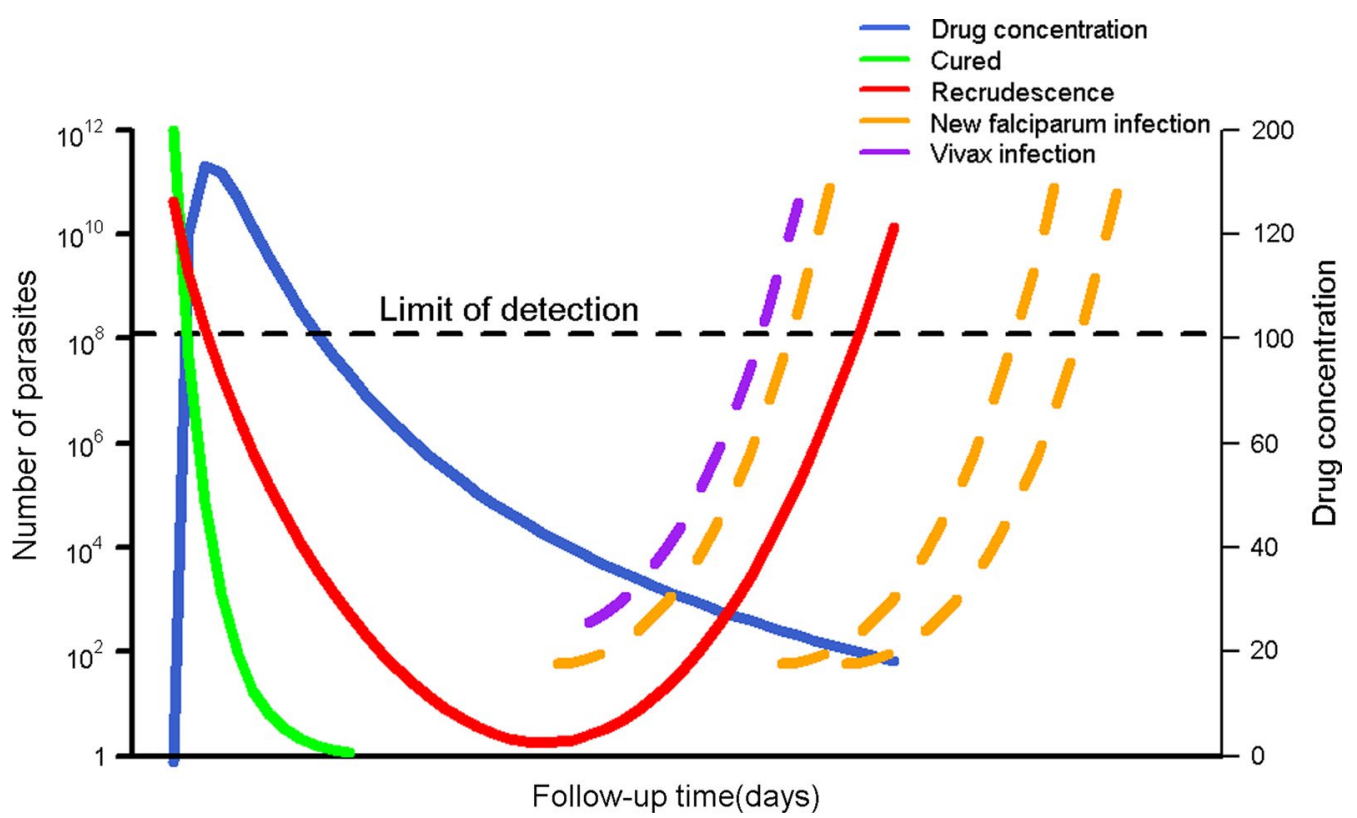

Fig. 1 Therapeutic responses post anti-malarial treatment. The blue line represents a hypothetical concentration versus time profile for an antimalarial drug administered orally. The green and red lines represent scenarios for parasite burden versus time profiles following treatment for an infection where all the parasites are completely killed resulting in cure (green) and an infection where parasites are initially killed by high drug levels but with drug levels below the minimum inhibitory concentration (MIC), net parasite growth results in subsequent recrudescence (red). The purple and orange lines represent parasite-time profiles for new infections; either an infection due to a new parasite of the same species (orange) or an infection with a Plasmodium vivax parasite (purple) during the follow-up. The left y-axis is for parasite density, and the right y-axis shows drug levels at hypothetical units. The horizontal black line represents the microscopic limit of detection for parasites. The maximum number of parasites a human body can contain is $10^{12}$ (Adapted from White-2002 [1])

Anti-malarial efficacy needs to be monitored routinely in endemic areas, so that early indications of drug resistance are recognized and malaria control activities can be revised accordingly [3]. Defining clinical efficacy of current treatment options is a key process in optimizing anti-malarial treatment policy. The World Health Organization (WHO) recommends that treatment efficacy corrected by polymerase chain reaction (PCR) genotyping (defined as 1 minus the risk of recrudescence at day 28) should be at least $90 \%$ for existing anti-malarials, and that novel regimens should achieve greater than $95 \%$ efficacy to be considered suitable as a first line treatment [4]. However, the classification of treatment outcomes based on PCR-genotyping is known to be vulnerable to its sensitivity, the resolution limits for detecting the differences in the allelic variants of the parasites, the definition used, the number of markers used, the transmission settings, and the genetic diversity of the markers used for sequencing and allele frequencies in different populations [5-8]. In areas of low transmission, multiplicity of infection (MOI) is low (in contrast to the areas of high transmission) [9] and the probability of the pre- and post-treatment alleles being the same due to chance is very small leading to a low misclassification risk for the PCR genotyping. Failure in collecting either the pretreatment or recurrent parasite DNA isolate will result in missing outcomes. Sometimes, the PCR technique is unable to discriminate recrudescences from new infections due to unsuccessful amplification of DNA or due to failure to interpret the results leading to indeterminate cases. Uncertainty in genotyping procedures leading to misclassification of outcomes has been well studied in anti-malarial literature and outcome classification is vulnerable to the algorithm used and transmission intensity $[8,10]$. In addition, study design, the presence of attrition bias, duration of follow-up and the choice of statistical methods to address these confounding factors can have a profound influence on the derived efficacy estimates [11-14].

In this review, the evolution of the methods for defining anti-malarial drug efficacy since the 1960s and the key statistical approaches currently available are documented. Challenges associated with these statistical methods and how they apply to standalone efficacy trials and comparative drug studies are discussed. 


\begin{tabular}{|c|c|c|}
\hline Host & Drug & Parasite \\
\hline Immunity & Pharmacokinetic-pharmacodynamics profile & Parasite biomass \\
\hline Age & Drug-drug interactions & Sequestration (a) \\
\hline Pregnancy & Dosing strategy & Mixed infections \\
\hline Co-morbidities & Duration of treatment & Staging (b) \\
\hline Pharmacogenomics & Tolerability & Synchronicity (c) \\
\hline Treatment seeking behaviour & Drug quality & Polyclonality \\
\hline Adherence & Drug formulations & Resistance \\
\hline History of drug use & & \\
\hline
\end{tabular}

Fig. 2 Determinants of in vivo response to anti-malarial treatment. ${ }^{a}$ The process by which infected erythrocytes containing mature parasites adhere to the microvasculature. Their removal of from the circulation results in the peripheral parasite count being an underestimate of the true parasite biomass. ${ }^{b}$ The developmental stage of the parasite. The artemisinin compounds have the broadest stage specific action against the parasite. ${ }^{c}$ Simultaneous rupture of hepatic schizonts result in a uniform stage distribution of the parasite

\section{Methods for estimating anti-malarial efficacy for a single treatment}

The WHO released its first standardized protocol for assessing in vivo efficacy against Plasmodium falciparum malaria in 1965 , primarily to monitor chloroquine resistance which had been identified a few years earlier $[15,16]$. This early protocol was revised twice, first in 1967 and then again in 1972, recommending that patients be kept in a mosquito free environment to prevent new infection [17]. These early protocols focused on parasitological outcomes, such as a recurrent peripheral parasitaemia and time to parasite clearance. In the subsequent revisions, methodologies remained largely the same until 1996, when the focus shifted from parasitological response to adequate clinical response [18]. The latter was defined as patients without clinical disease and included those with recurrent parasitaemia but without symptoms. The $1996 \mathrm{WHO}$ protocol focused on parasite clearance assessed on day 14 downgrading the importance of a longer follow-up used for characterizing anti-malarial efficacy-a recommendation retrospectively found to be inadequate [11]. The recommendation were revised again in 2009, when a composite endpoint of both parasitological and clinical assessment was adopted and this was defined as "adequate clinical and parasitological response (ACPR)" [3]. Whilst the earlier guidelines focused on the broader aspects of malarial chemotherapy, the later guidelines have focused more on the methodological aspects of clinical studies. The evolution of these documents and relevant methodical reviews is presented in Fig. 3.

Two approaches have been used in deriving efficacy estimates in anti-malarial efficacy studies: (i) the calculation of the proportion of patients cured within a specified period of follow-up (this proportion is often referred to as the "cure rate") and (ii) survival analysis, which provides a cumulative probability of cure. The term "cure rate" statistically speaking is misleading since this is not a rate, but a point estimate of risk at a predefined time point. The proportion cured is usually estimated using a per-protocol (PP) and intention to treat (ITT) approaches. In PP approach, the proportion cured is derived from all patients followed until treatment failure or a set period of time, excluding those with protocol deviations, those who develop new infection or who are lost to follow-up. Whilst relatively easy to calculate, this approach ignores valuable information provided by the patients who experience protocol deviations or are lost to follow-up. Patients failing treatment are more likely to become symptomatic and seek retreatment and thus be detected passively. Whereas patients who are cured are more likely to tire from active detection and be lost to 


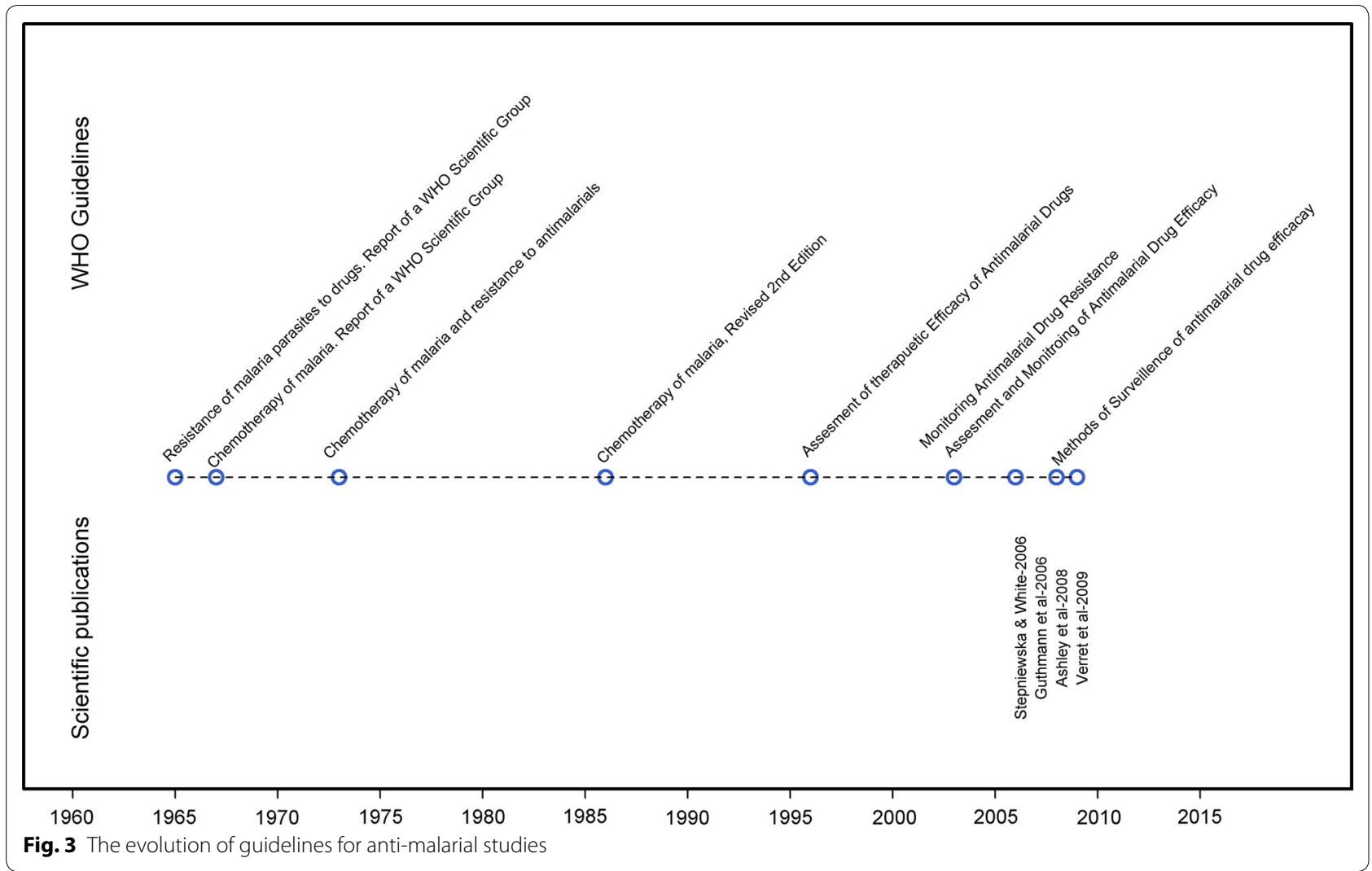

follow up-these attrition biases result in an underestimation of treatment efficacy [13]. In a more conservative analysis using an ITT approach, the evaluable population includes all patients enrolled in the study, but patients who are lost to follow-up or who experience protocol deviations are considered as treatment failures. This will underestimate treatment efficacy (and overestimate clinical resistance). This definition of ITT used within the context of anti-malarial studies differs from the standard terminology used in randomized controlled trials, in which ITT generally means that all study participants are included in the analysis as part of the groups to which they were randomized regardless of whether they completed the study or not. Assigning patients who do not complete follow-up the worst possible outcome ensures that derived estimates represent the "worst case scenario".

Survival analysis using Kaplan-Meier (K-M) method provides an alternative strategy. This approach maximizes the information available from each patient, thereby increasing the precision of the estimates [12, 13]. The $\mathrm{K}-\mathrm{M}$ estimates of the cumulative probability of cure are usually reported at day 28 and for studies with longer follow-up duration the estimates at the end of the study (e.g. day 42 and 63) are also presented. The complement of $\mathrm{K}-\mathrm{M}$ (1 minus $\mathrm{K}-\mathrm{M})$ is frequently used to derive estimate of the cumulative proportion of treatment failure. In the $\mathrm{K}-\mathrm{M}$ approach, patients who do not fail treatment during the study period and do not complete follow up for any reasons are included in the analysis until the time of last recorded visit when they are "censored". Patients who are censored are considered to be at the same risk of experiencing the event of interest as those who continue to be followed, i.e. the censoring is uninformative [19]. Although survival analysis has long been used in other disease areas and in one anti-malarial study in 1995 [20], it was only widely considered for deriving anti-malarial efficacy in 2001 [21] and adopted into the WHO guidelines in 2003 [17]. Stepniewska and White provided a further assessment on the methodological approaches used in anti-malarial studies, and strongly advocated the use of $\mathrm{K}-\mathrm{M}$ method [12]. A tutorial on deriving efficacy estimates using the $\mathrm{K}-\mathrm{M}$ survival approach is presented in Additional file 1: Section A.

Several reports have compared the use of PP approach and $\mathrm{K}-\mathrm{M}$ survival analysis in deriving anti-malarial efficacy. Guthmann et al. pooled datasets from 13 trials $(\mathrm{n}=2576)$ to examine the discrepancies in derived estimates when PP and $\mathrm{K}-\mathrm{M}$ approach were used [14]. Overall $6 \%$ of the samples were lost by day 28 using K-M analysis when indeterminate outcomes were excluded 
and new infections were treated as treatment success. In contrast, there was a $25 \%$ reduction in sample size using PP approach. The risk of recrudescence estimates were lower with the $\mathrm{K}-\mathrm{M}$ method and the risk differences ranged from -2.3 to $2.3 \%$ when indeterminate cases were excluded. Similar finding was reported by Ashley et al., where the use of PP method was associated with a $34 \%$ reduction in sample size as opposed to $<10 \%$ reduction when survival analysis was used [22]. In a pooled analysis of 29 clinical trials from Africa and Asia carried out by Verret and colleagues, the PP method consistently overestimated the risk of treatment failure compared to the $\mathrm{K}-\mathrm{M}$ approach (median difference: $1.7 \%$, range $0-30.9 \%$ ) and the magnitude of overestimation was proportional to the incomplete follow-up [13]. The authors of these studies recommended the use of $\mathrm{K}-\mathrm{M}$ analysis, as this minimized the loss of information and made the maximum use of the data.

Since the K-M approach was being increasingly recommended for deriving efficacy estimates, Price et al. provided a classification table for different possible outcomes (see Table 3 of the article) [23]. The WHO guideline published in 2009 further recommended the use of $\mathrm{K}-\mathrm{M}$ method for deriving anti-malarial efficacy where patients who are lost to follow-up or who develop a new infection during the follow-up period or any other deviations are censored on the day of their last observation in the trial (Table 1) [3]. However, there are several pitfalls and challenges associated with $\mathrm{K}-\mathrm{M}$ approach that need to be considered [24], and these are addressed in sections to follow, with example from a large multi-centre study carried out in Uganda.

\section{Example dataset}

Data from a randomized control trial which compared three anti-malarial regimens in four different sites in Uganda from 2002 to 2004 was used as a motivating example [25]. Briefly, 2160 patients aged 6 months or older were randomized to one of the three treatment arms: chloroquine + sulfadoxine-pyrimethamine $(\mathrm{CQ}+\mathrm{SP})$, amodiaquine + sulfadoxine-pyrimethamine $(\mathrm{AQ}+\mathrm{SP})$ or amodiaquine plus artesunate $(\mathrm{AS}+\mathrm{AQ})$. The primary endpoint was the risks of parasitological failure either unadjusted or adjusted by PCR genotyping at the end of the study follow-up on day 28 . The study was standardized using the WorldWide Antimalarial Resistance Network (WWARN) clinical protocol [26] and hence the estimates reported in the original article are slightly different to the estimates reported here.

\section{Challenges in estimating efficacy for a single treatment The presence of competing endpoints}

In an anti-malarial trial of uncomplicated $P$. falciparum malaria, the primary endpoint is the risk of recurrence due to reappearance of the same parasite which caused the initial infection (recrudescence). However, patients can experience new infections with $P$. falciparum or other species such as Plasmodium vivax during the ensuing weeks (Fig. 1). Such alternative outcomes which can preclude the occurrence of recrudescence are referred to as competing risk events [24]. When studies are conducted in a malaria endemic setting these competing risk events can sometimes occur in over $30 \%$ of patients [13]. Once a patient experiences competing events before the end of the study follow-up, recrudescence can no longer

Table 1 Assigning outcomes for estimating treatment efficacy under current recommendations. Source: WHO-2009 [3]

\begin{tabular}{|c|c|c|}
\hline End-point for day $X(X=28$ or 42$)$ & $\begin{array}{l}\text { Cumulative success or failure probability } \\
\text { (Kaplan-Meier analysis) }\end{array}$ & Proportion (per-protocol analysis) \\
\hline Adequate clinical and parasitological response at day $X$ & Success & Success \\
\hline Early treatment failure & Failure & Failure \\
\hline Late clinical failure before day 7 & Failure & Failure \\
\hline \multicolumn{3}{|c|}{ Late clinical failure or late parasitological failure on or after day 7} \\
\hline Falciparum recrudescence & Failure & Failure \\
\hline Falciparum reinfection & Censored day of reinfection & Excluded from analysis \\
\hline Other species with falciparum recrudescence & Failure & Failure \\
\hline Other species infection & Censored day of infection & Excluded from analysis \\
\hline Undermined or indeterminate PCR & Excluded from PCR-corrected analysis & Excluded from analysis \\
\hline Loss to follow-up & Censored last day of follow-up according to timetable & Excluded from analysis \\
\hline Withdrawal and protocol violation & $\begin{array}{l}\text { Censored last day of follow-up according to timetable } \\
\text { before withdrawal or protocol violation }\end{array}$ & Excluded from analysis \\
\hline
\end{tabular}


be observed as the first event. The presence of competing events changes the number of people remaining at risk of recrudescence and consequently the probability of true treatment failure. In such situations, the overall probability of failing due to recrudescence should be estimated by accounting for the treatment failures due to recrudescence and also recurrence due to the competing events [24].

The $\mathrm{K}-\mathrm{M}$ method makes a fundamental assumption of independent (non-informative) censoring, i.e. patients who are censored have the same risk of observing the outcome as those who are still being followed-up. When a patient experiences new infections, censoring is no longer non-informative (as they will be retreated) and in such situations the use of $\mathrm{K}-\mathrm{M}$ leads to an upwards biased estimate of treatment failure [24, 27, 28]. Despite this limitation, the complement of $\mathrm{K}-\mathrm{M}$ estimate (i.e. 1 minus $\mathrm{K}-\mathrm{M}$ ) is commonly used to derive the cumulative probability of failure in anti-malarial studies. An alternative approach in the presence of competing risk events is the derivation of the Cumulative incidence function (CIF) as proposed by Kalbfleisch and Prentice [29]. CIF estimates the risk of failing from a specific cause at any time between enrolment $\left(t_{0}\right)$ and the time point of interest $\left(t_{x}\right)$ and this takes into account the failures from other causes (see Additional file 1: Section A for a tutorial).

A comparison of the $\mathrm{K}-\mathrm{M}$ method and CIF is illustrated using example data from Tororo site in Uganda, an area of high transmission where new infections during follow up were frequently observed [25]. Data from 166 patients treated with CQ + SP were originally analysed using the $\mathrm{K}-\mathrm{M}$ method and were reanalysed using the CIF approach using cmprsk package in R software (Additional file 1: Section B) [30]. The estimate of cumulative probability of recrudescence on day 28 was 0.376 [95\% CI 0.264-0.470] using the K-M approach (Fig. 4a) [25], compared with 0.265 [0.199-0.331] calculated using the $\mathrm{CIF}$ approach. The corresponding probabilities of reinfections by day 28 were 0.761 [0.667-0.828] using $\mathrm{K}-\mathrm{M}$ and 0.654 [0.584-0.723] using CIF. These values represent an absolute overestimation of 0.11 for both recrudescence and new infections. In relative terms, this represents an overestimation of treatment failure using $\mathrm{K}-\mathrm{M}$ approach by $41.8 \%$ for recrudescence and $24.4 \%$ for new infections. Such overestimation is particularly relevant to new treatment regimens as increasingly high efficacy (>95\%) is demanded for a drug to be considered as a first line therapy. This bias is likely to be worse for regimens which offer only short post treatment prophylaxis in high transmission areas, as new infections are more likely to occur, than for regimens which provide longer protection; this difference in the incidence of new infections over the follow-up period has implications for trials comparing different anti-malarials (comparative trials section to follow). Another challenge with the $\mathrm{K}-\mathrm{M}$ method is that the sum of individual $\mathrm{K}-\mathrm{M}$ estimates for different events
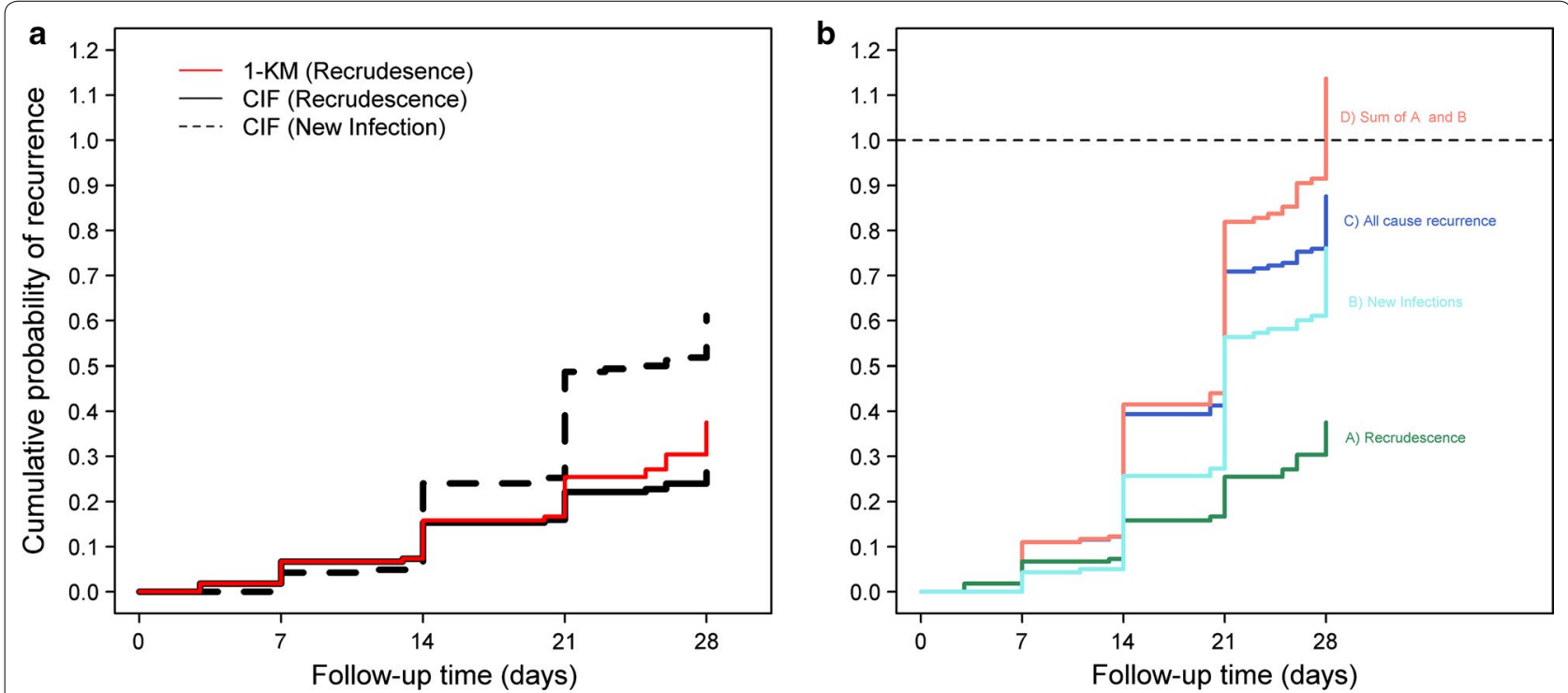

Fig. 4 Overestimation of failure using complement of K-M method in Tororo dataset [25]. a Cumulative probability of failure due to recrudescence derived using Kaplan-Meier approach (red line) and using Cumulative Incidence Function (solid black line), which accounts for the presence of competing risks (dotted black line). b Estimates of the cumulative probability of recurrences for recrudescence (green), new infections (light blue) and overall recurrences (dark blue line) using $\mathrm{K}-\mathrm{M}$ method. The sum of the probabilities for recrudescence and new infection is presented as the pink line and exceeds the value of 1 at 28 days of follow-up 
(e.g. recrudescence and new infection in malaria studies) can exceed the $\mathrm{K}-\mathrm{M}$ estimate for the composite endpoint of overall recurrence. The sum of the cumulative probability of recurrence estimated using $\mathrm{K}-\mathrm{M}$ approach for recrudescence and new infection on day 28 was $113.7 \%$, whereas the all cause recurrence estimate was only $87.6 \%$ (Fig. 4b). In such situations, the interpretation of $\mathrm{K}-\mathrm{M}$ estimate as a probability is no longer valid as the sum exceeds $100 \%$.

\section{Follow-up data are interval-censored}

In anti-malarial studies, active surveillance is generally conducted weekly with follow-up usually scheduled daily for the first 3 days and weekly from day 7 until 28, 42 or 63 days. Thus recurrent parasitaemia, particularly in patients who are asymptomatic, is actively detected and commonly occurs on pre-scheduled follow-up timepoints. However, the true timing of microscopically patent positive recurrent parasitaemia is often in between the times of observation, and this gives rise to intervalcensored failure times in anti-malarial studies. Ignoring such intervals might lead to an under or overestimation of failures at a given time point, especially when it is assumed that failures occur at the beginning or the end of the interval, and the magnitude of the bias tends to be accentuated as the length of the interval gets larger [31]. Despite this, interval censored data are analysed frequently using $\mathrm{K}-\mathrm{M}$ method in an ad hoc approach of assuming the failures observed at pre-scheduled visits as the true failure time. Interval censored methods are now part of standard statistical packages and there exists substantial literature on survival estimation and regression methods [32-35]; the algorithm proposed by Turnbull being the most commonly used [32].

The $\mathrm{K}-\mathrm{M}$ estimates derived using the interval censored method for the Uganda data were similar to the $\mathrm{K}-\mathrm{M}$ estimates generated by ignoring the interval censoring, and the results and $\mathrm{R}$ script for analysis is presented in Additional file 1: Section C.

\section{Multi-centre trials}

Clinical surveillance studies sometimes enrol patients from more than one centre in order to achieve adequate sample size and sample geographically distinct populations, thus increasing the generalizability of the results. Data are often pooled across sites to get an overall estimate of drug efficacy, but this requires careful consideration. Although samples from different sites are assumed to be independent, there may be heterogeneity in censoring patterns, attrition rates, patient demographics, and transmission intensity. The overall cured proportion at a fixed time point can be computed by combining estimates from each of the sites using standard meta-analysis methods for pooling proportions; the approach of DerSimonian and Laird's being the most common [36]. However, synthesizing survival curves across sites is challenging. The Cochrane Handbook for Systematic Reviews of Interventions comments on the difficulty in presenting a pooled estimate of $\mathrm{K}-\mathrm{M}$ from different studies as follows [37]:

\section{"Kaplan-Meier plots for all pooled participants across trials in a meta-analysis have previously been presented in medical journals. This practice breaks with the principle of comparing like with like. For this reason, until further discussions have taken place the Statistical Methods Group is unable to recommend inclusion of such plots in Cochrane reviews."}

The Cochrane statement is regarding the presentation of the survival curve in a meta-analysis; and this is also relevant for multi-centre studies. However, no specific guidance is provided regarding presentation of point estimates of $\mathrm{K}-\mathrm{M}$ at specified time points and there exists no consensus among researchers on the best approach to synthesize survival estimates across studies/sites, neither for the aggregate meta-analysis nor for individual patient data meta-analysis. It is common to perform the analysis in a one-step approach where raw data from several sites are pooled as if they came from a single site (naïve approach) and present an overall $\mathrm{K}-\mathrm{M}$ estimate without considering the multi-centric nature of the data, an approach recommended by Srinivasan and Zhou provided the data from several sites (studies) are independent [38]. However, due to heterogeneity across centres, such approach can result in a treatment appearing to be beneficial after pooling data from several sites, in situations where the reverse is in fact true [39]. Recently, Comberscure et al. proposed a method to pool the $\mathrm{K}-\mathrm{M}$ estimates from several studies at specific time point [40]. Using this approach, $\mathrm{K}-\mathrm{M}$ and number of patient at risk are derived for each site at a time-point of interest. DerSimonian and Laird's $(D+L)$ method is then applied after carrying out an arc-sine transformation of the survival to obtain a pooled estimate of the $\mathrm{K}-\mathrm{M}$. This is available through MetaSurv package on open source R software [41].

The meta-analytic approach proposed by Comberscure et al. has been illustrated with the example dataset on AS + AQ arm [40]. The K-M estimates for each of the study sites were extracted at the all the pre-scheduled visits (days 1, 2, 3, 7, 14, 21 and 28) including the number of people at risk. The pooled $\mathrm{K}-\mathrm{M}$ estimates estimated using the naïve approach D + L's approach are presented in Table 2. The $\mathrm{R}$ script for performing analysis is presented in Additional file 1: Section D. In this example, the 
Table 2 Pooled Kaplan-Meier estimates for recrudescence using naïve and metasurv approaches for AS + AQ [25]

\begin{tabular}{lll}
\hline Day & \multicolumn{2}{l}{ Pooled Kaplan-Meier estimates } \\
\cline { 2 - 3 } & Naïve approach ${ }^{\mathbf{a}}$ & $\mathbf{D}+$ L approach meta-analysis \\
& $\mathbf{b}$ \\
\hline 7 & 1.000 & $0.994[0.987-1.000]$ \\
14 & $0.994[0.989-1.000]$ & $0.989[0.979-0.998]$ \\
21 & $0.949[0.933-0.966]$ & $0.943[0.916-0.970]$ \\
28 & $0.918[0.895-0.941]$ & $0.909[0.873-0.948]$ \\
\hline
\end{tabular}

a Kaplan-Meier estimates were estimated assuming the data came from one single study. Patients with new infections, indeterminate outcomes and lost to follow-up were censored when deriving the $\mathrm{K}-\mathrm{M}$ estimates for recrudescence failures

${ }^{b}$ Kaplan-Meier estimates and associated number of patients at risk were extracted on pre-scheduled follow-up days 1, 2, 3, 7, 14, 21, and 28. The estimates were pooled using MetaSurv package in $\mathrm{R}$ (Additional file 1: Section D). I squared statistic for heterogeneity $=0 \%$

pooled estimates at day 28 were similar between the two approaches; with the confidence intervals being wider for the estimates derived using D + L's approach. However, this approach needs to be used with caution as the bias in estimates could be high when sample size per site is small or when the events are rare [40]. In such situation, an alternative approach could be to apply the $\mathrm{D}+\mathrm{L}$ procedure after complementary log log transformation $\{\log (-\log ())$.$\} of the survival function. This approach$ has the desired statistical property of giving correct coverage probability for the estimated 95\% confidence interval of the survival estimate based on as few as 25 observations [42].

\section{Challenges specific to comparative trials}

To investigate suitable alternative treatment regimens, a comparative randomized clinical trial is required and these comparative trials raise further difficulties in the analyses and interpretation of the data.

\section{Comparison of anti-malarial drugs with different pharmacokinetic profiles}

Anti-malarial drugs with different elimination halflives may vary considerably in the period of time during which peripheral parasite growth is suppressed. Since post treatment prophylaxis reduces the risk of new infection and delays the timing of recurrent parasitaemia, for drugs with similar efficacy the comparative results may be biased against the drug with the shorter half-life. Conversely, a new drug which has a long elimination halflife may result in the false impression of good efficacy. Consider dihydroartemisinin-piperaquine (DP) and artemether-lumefantrine (AL). Piperaquine is a slowly eliminated regimen with a terminal elimination half-life of 13.5-28 days and will suppress parasitic growth for a far longer period compared to lumefantrine, which has an elimination half-life ranging from 1.4 to 11.5 days [4]. Patients treated with DP will therefore encounter fewer recrudescences/new infections during the first 28 days of the follow-up and thus comparison of efficacy at day 28, will be biased against artemether-lumefantrine (AL; see Fig. 5 for an illustration of this bias). For example, in a longitudinal study conducted in Uganda, the median time to recrudescence was 21 days (range 21-50 days) in the AL arm compared to 42 days (range 33-51 days) in the DP arm [43].

Ideally, the comparison overall efficacy of anti-malarial regimens should be carried out at a time, when antimalarial drug concentrations cease to suppress parasite growth (i.e. fall below the minimum inhibitory concentration-MIC) and after allowing the time for the parasites to reach the limit of detection. If the drug concentrations fall below MIC on day 18, and assuming 10 parasites are circulating in the blood, these parasites will reach the threshold for detection $\left(\sim 10^{8}\right.$ parasites $)$ in 7 parasite life cycles, which is approximately 14 days assuming an efficient multiplication of tenfold per cycle and a parasitic developmental cycle of $48 \mathrm{~h}$. In this scenario recrudescences begin to appear by day 33 . Under the same assumption, a drug which provides more prolonged prophylaxis (e.g. with drug concentrations in plasma falling below than MIC on day 31) will result in recrudescent parasites reaching the limit of detection on day 45 . Hence, comparing these two drugs on day 42 will result in a biased conclusion. The comparative efficacy should account, therefore, for differences in the pharmacokinetic profile of the drugs. This is further confounded by transmission setting, which determines the risk of new infection (a competing risk event). Characterization of the duration of follow-up required to appropriately capture the treatment failures would provide a basis for comparison and this is currently being investigated [44].

Comparing survival estimates at a fixed point in time in a single centre study Comparison of survival curves is usually carried out using the log-rank test. In competing risk analysis situation, such comparison is made using Gray's test [45]. The log-rank test uses information throughout the study follow-up period with equal weights given to failures at all time points. This is the most powerful test under the assumption of proportional hazards. Intersection of two survival curves may be indicative of non-proportional hazards and the log-rank test will fail to pick up differences. In such situation Gehan's test and non-parametric tests such as Kolmogorov-Smirnov and Cramer-von Mises types of tests may be used [42]. Which of these two tests (log-rank or Gray's test) remains the appropriate approach has gathered considerable interest 


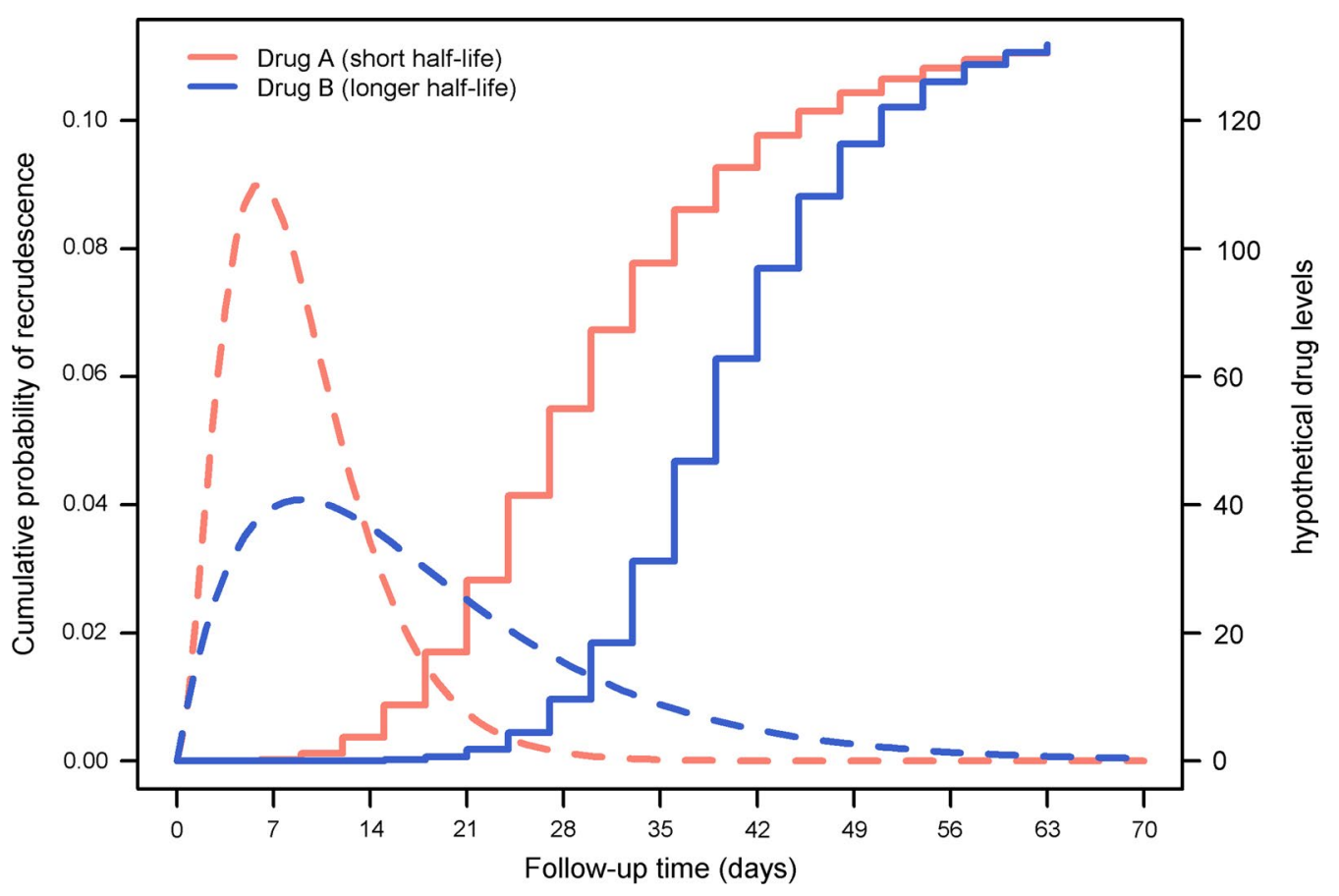

Fig. 5 Cumulative failure estimates for drugs with different terminal elimination half-lives. The solid line represents K-M cumulative failure probabilities and the dotted line represents the drug levels with hypothetical units presented on the right $y$-axis. Drug B (blue) has a longer elimination half-life and the recrudescent failures are more patent after day 21 compared to drug A (red) with a short half-life and recrudescences being observed after day 7

in statistical literature. It has been suggested that if the interest is in understanding the biological mechanism (e.g. how a treatment affects recrudescence), the log rank test is considered appropriate and when a researcher is interested is answering if subjects receiving a particular drug are more likely to fail (e.g. experience recrudescence by the end of the study), the comparison of CIF through Gray's test is considered appropriate [24, 46, 47].

In addition, when comparing two anti-malarial regimens (e.g. AL and DP) it appears to be more relevant to focus on the overall proportion of failures observed during the follow-up time. Consequently, an alternative approach test is needed which allows comparison of the cumulative Kaplan-Meier estimates at a specific time point. Such a test can be constructed from the difference of complementary log-log transformed $\mathrm{K}-\mathrm{M}$ estimates at a specified time point and the appropriately estimating the standard error for this difference [42].

Let $\hat{S} 1(t)$ and $\hat{S} 2(t)$ be the two survival estimates and $\delta=\{\ln (-\ln (\hat{S} 1(t)))-\ln (-\ln (\hat{S} 2(t)))$ be the difference between these two estimates on complementary log-log scale. The $X^{2}$ test statistic for the comparing the difference in two estimates is given by [42]:

$$
X^{2}=\frac{\{\delta\}^{2}}{\operatorname{Var}(\delta)}
$$

where $\operatorname{Var}(\delta$ ) denotes variance estimate of $\delta$ (see Additional file 1: Section $\mathrm{E}$ for details of derivation and $\mathrm{R}$ associated script). This statistic has an approximate Chi squared distribution in 1 degree of freedom. The approach recommended by Klein et al. [42] (referred as Klein's test onwards) has been illustrated using example data 2 provided in Additional file 1: Section E. There was a significant difference between drug $A$ and drug $B$ on day 42 based on log-rank test $\left(X^{2}=4.30\right.$, $\mathrm{p}=0.038)$. Using Klein's test, there were no difference in the $\mathrm{K}-\mathrm{M}$ estimates on day $42\left(\mathrm{X}^{2}=0.266\right.$, $\mathrm{p}=0.606$ ) (Fig. 6).

Comparing survival estimates at a fixed point in time in multicentre trials Klein's test to compare survival estimates at a fixed point in time can be extended to multicentre studies (Additional file 1: Section E) [42]. The result of the Klein's test and the stratified log-rank test for comparing AQ + SP against AS + AQ were similar and are presented in Table 3. 


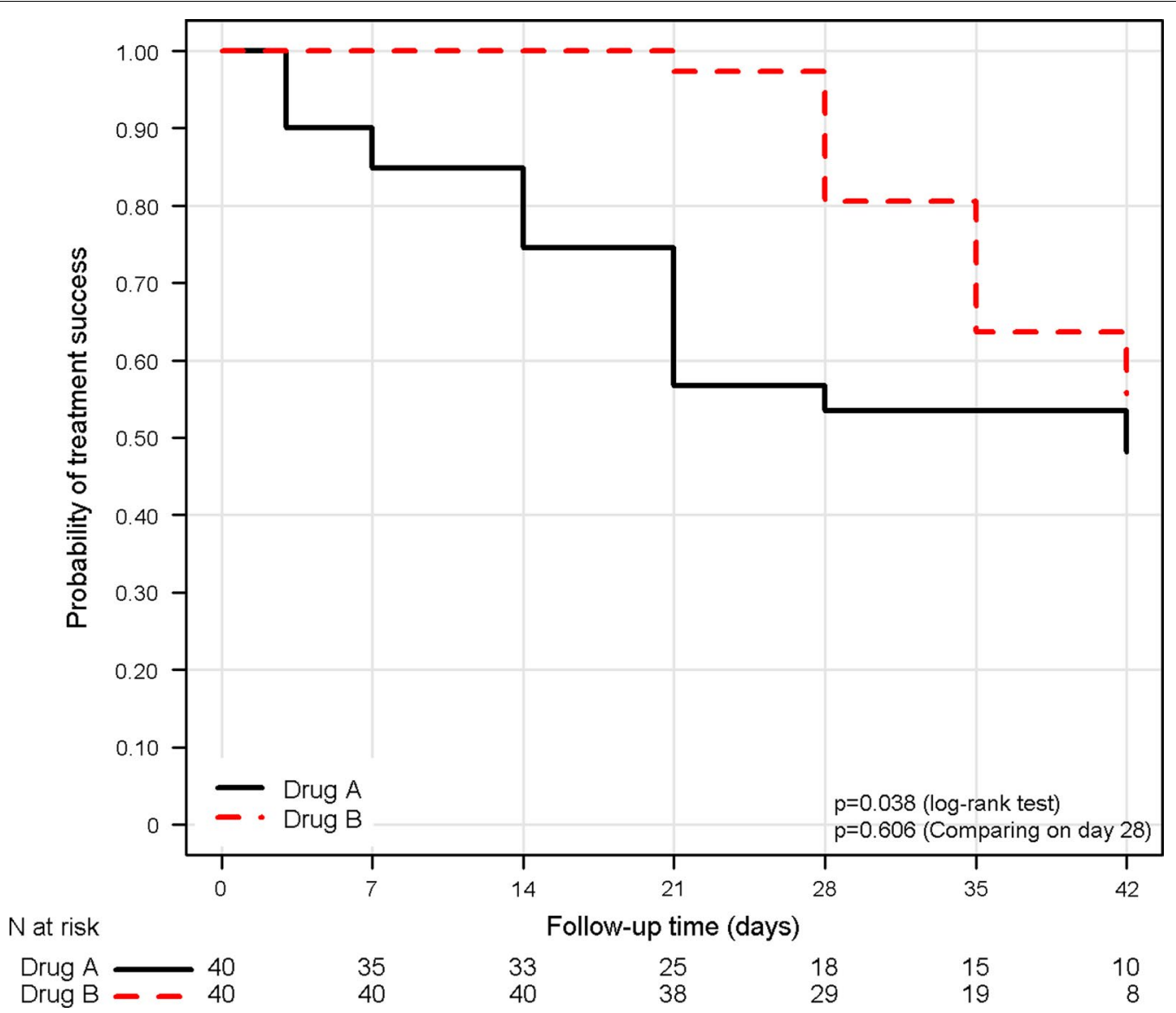

Fig. 6 Comparison of survival estimates at fixed point in time

\section{Non-inferiority designs}

Historically clinical trials have been designed to determine the superiority of a new regimen against that of a failing drug [48]. This approach is useful when the efficacy of the standard regimen has already reached unacceptable levels. However, when comparing highly effective regimens it is often unfeasible to demonstrate the superiority since the required sample size will be

Table 3 Comparing $\mathrm{K}-\mathrm{M}$ at fixed point in time

\begin{tabular}{llllll}
\hline Site & $\begin{array}{l}\text { Chi squared test for com- } \\
\text { paring K-M at a fixed } \\
\text { time point (day 28) }\end{array}$ & & $\begin{array}{l}\text { Log-rank test } \\
\text { for comparing } \\
\text { (Klein's test [42]) }\end{array}$ & & \multicolumn{2}{l}{$\begin{array}{l}\text { whole curve (day 0 } \\
\text { to day 28) }\end{array}$} \\
\cline { 2 - 3 } \cline { 5 - 6 } & $\boldsymbol{X}^{\mathbf{2}}$ & $\boldsymbol{p}$ value & & $\boldsymbol{X}^{\mathbf{2}}$ & $\boldsymbol{p}$ value \\
\hline Tororo & 1.35 & 0.246 & & 2.00 & 0.158 \\
Arua & 2.72 & 0.099 & & 2.20 & 0.142 \\
Jinja & 6.18 & 0.013 & 6.40 & 0.011 \\
Apac & 0.91 & 0.340 & 1.10 & 0.290 \\
Stratified test & 4.98 & 0.026 & 4.90 & 0.026 \\
\hline
\end{tabular}

extremely large. In this scenario a non-inferiority design is often adopted [12]. The primary objective of a noninferiority trial is to demonstrate that an investigational drug regimen is not clinically inferior ("is no worse") to the current standard of care. This can be demonstrated by showing the two-sided $95 \%$ confidence interval (CI) for treatment difference is likely to lie above a lower margin of clinically acceptable differences $(\Delta)$. Currently, there exists no recommendation regarding the optimal $\Delta$ margin for comparative studies. The US Food and Drug Administration (FDA) requires construction of twosided $95 \%$ confidence interval for the difference in cure with a pre-specified $\Delta$ for the per-protocol and modified ITT population to demonstrate non-inferiority [49]. For anti-malarial studies, Borrmann et al. recommend using a non-inferiority margin of $5 \%$ units (or, its equivalent as hazards ratio unit) for phase III trials provided that the cured proportion remain above 90\% [48]. Since antimalarial efficacy with an ACT regimen is invariably close to or greater than $95 \%$, a non-inferiority margin of $5 \%$ can be regarded as a reasonable choice. Smaller margin will require a much larger sample size, which has immediate 
implication on cost and resources. A larger margin will lead to a smaller sample size but would not guarantee that the efficacy of the comparator is greater than $90 \%$.

Currently the demonstration of non-inferiority is widely based on the cured proportion. Since the K-M method is the recommended statistical approach for deriving anti-malarial efficacy, any demonstration of non-inferiority should be based ideally on differences in the $\mathrm{K}-\mathrm{M}$ estimates. This can be carried out a fixed point in time or can be based on the whole curve.

Demonstrating non-inferiority based on $K-M$ estimates at a fixed point in time The hypothesis of non-inferiority at a fixed time point (e.g. day 28) can be tested using the $\mathrm{K}-\mathrm{M}$ estimates. Suppose $\Delta$ is the margin for non-inferiority. The $95 \% \mathrm{CI}$ for the difference of two $\mathrm{K}-\mathrm{M}$ estimates can be constructed by adding individual variance of the two $\mathrm{K}-\mathrm{M}$ estimates [50]. For anti-malarial studies, Stepniewska and White have proposed the use of effective sample size for constructing 95\% CI for differences in $\mathrm{K}-\mathrm{M}$ estimates and this can be easily applied for any trial [12]. The effective sample size is calculated by dividing the derived $\mathrm{K}-\mathrm{M}$ estimates at a given time point by the number of patients who reached the end of the study without any deviations or treatment failure (see Additional file 1: Section F for a worked out example).

Demonstrating non-inferiority based on relative risk measure Under the proportional hazards assumption, the non-inferiority between regimens can be tested based on the relative risk measure (e.g. hazards ratio). Let $\hat{S} 1(t)$ and $\hat{S} 2(t)$ be survival estimates for the new and standard treatment regimens respectively at time $t$. Let $\gamma$ be the corresponding relative risk of failure for new treatment compared to the standard regimen (estimated as hazards ratio from Cox's model) and let $\Delta=\hat{S} 1(t)-\hat{S} 2(t)$ be the non-inferiority margin. Under the assumption of proportional hazards [51],

$$
\hat{S} 1(t)=[\hat{S} 2(t)]^{\gamma} \Rightarrow \gamma=\frac{\ln (\hat{S} 1(t))}{\ln (\hat{S} 2(t))}
$$

The upper limit for non-inferiority based on relative risk then can be derived based entirely on the efficacy of the reference (standard) arm as:

$$
\gamma=\frac{\ln (\hat{S} 2(t)-\Delta)}{\ln (\hat{S} 2(t))}
$$

Non-inferiority is demonstrated if the $95 \%$ CI for the estimated hazards ratio remains below the

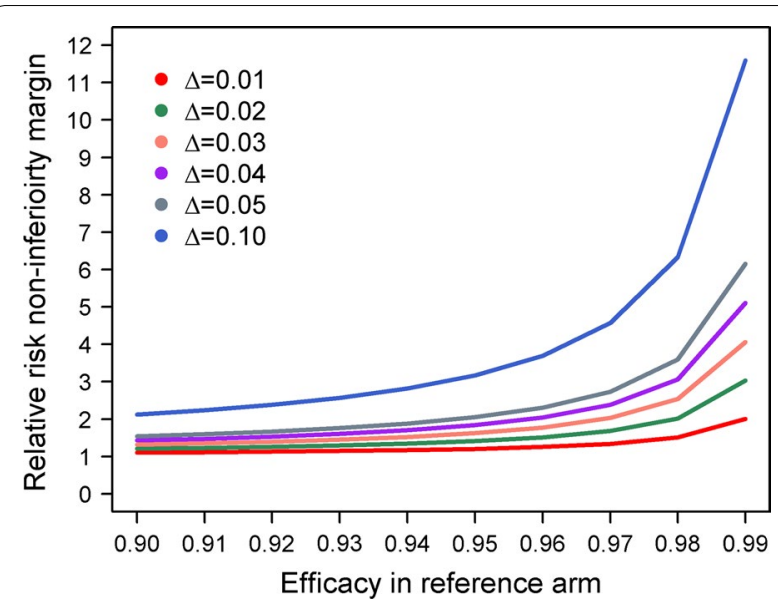

Fig. 7 Upper limit of non-inferiority margin based on the relative risk. Relationship between non-inferiority margin on the relative risk scale, and the margin on the survival difference (difference of two KaplanMeier estimates) scale, $\Delta$

non-inferiority limit on the relative risk scale [48]. Further recommendations on interpretation of the results of the non-inferiority tests for Phase III studies is provided elsewhere (see Table 1 of Bormann et al. [48]). Figure 7 shows the relative risk non inferiority margin corresponding to survival estimates $>90 \%$ in the reference treatment (Additional file 1: Section G). If 5\% difference is considered an appropriate non-inferiority margin in $\mathrm{K}-\mathrm{M}$ scale, then on relative risk scale, when the existing treatment has an efficacy of $97 \%$, the upper limit of the $95 \%$ CI for the derived hazards ratio for the new treatment should not exceed 2.7. One advantage of demonstrating non-inferiority on the relative risk scale as opposed to absolute differences in $\mathrm{K}-\mathrm{M}$ is that the influence of baseline covariates can be adjusted when deriving the hazards ratio using Cox's regression model as outlined by Tunes da Silva et al. [51]. However, one should be careful when performing such comparison between regimens with different half-lives and must validate the assumption of hazards being proportional. When the assumption of proportionality doesn't hold, an alternative approach is to construct the test based on the complementary $\log -\log$ transformation of the two survival estimates at time $t$. Logarithm of the hazard ratio corresponds to the difference between $\mathrm{K}-\mathrm{M}$ survival estimates on the complementary log-log scale. The 95\% confidence interval (on the complementary $\log \log$ scale) for this difference can be calculated using expression in Additional file 1: Section E. The estimated confidence interval can then be transformed into a relative ratio (hazard ratio) scale by exponentiation (a working example is presented in Additional file 1: Section $\mathrm{H})$. 
Table 4 Challenges in estimating antimalarial drug efficacy and possible alternatives

\begin{tabular}{|c|c|c|c|}
\hline Challenges & Current approach & Alternative approach & Software \\
\hline Competing risk event & Censored on the day of event [3] & Cumulative incidence function & $\begin{array}{l}\text { cmprsk package R [30] } \\
\text { (Additional file 1: Section B) }\end{array}$ \\
\hline Interval censoring & lgnored & Interval censored survival estimates & $\begin{array}{l}\text { survival package R [57] } \\
\text { (Additional file 1: Section C) }\end{array}$ \\
\hline K-M for multicentre studies & No specific recommendation & Use of meta-analysis approach [40] & $\begin{array}{l}\text { MetaSurv package R [41] } \\
\text { (Additional file 1: Section D) }\end{array}$ \\
\hline Comparing $\mathrm{K}-\mathrm{M}$ estimates & $\begin{array}{l}\text { Current comparison based on whole } \\
\text { survival curve (log-rank test) }\end{array}$ & $\begin{array}{l}\text { Comparison at a fixed point in time } \\
\text { based on complementary log-log } \\
\text { transformation [42] }\end{array}$ & $\begin{array}{l}\text { R script available as additional file } \\
\text { (Additional file 1: Section E) }\end{array}$ \\
\hline Demonstrating non-inferiority & Based on the cured proportion & $\begin{array}{l}\text { Based on the difference of two K-M } \\
\text { estimates after complementary log- } \\
\text { log transformation (or on hazards ratio } \\
\text { scale) }\end{array}$ & $\begin{array}{l}\text { R script available as additional file } \\
\text { (Additional file 1: Sections } F, G, H \text { ) }\end{array}$ \\
\hline
\end{tabular}

\section{Conclusions}

Significant resources have been spent to overcome recognized difficulties in estimating anti-malarial efficacy by standardizing methodological procedures and these have facilitated the monitoring of anti-malarial drug resistance over time and geographical location. However, even a robust design and analysis requires that the derived point estimates of efficacy are interpreted with caution and the confidence interval around such point estimates should be given equal importance when interpreting the result of a study. The study design, transmission setting, laboratory and genotyping procedures, patient demographics and adherence to protocols all need to be considered. Uncertainty associated with PCR genotyping can be ameliorated to a certain extent if allelic distribution and clonality of infection in the study population is known. This allows for adjustment of pre and post treatment alleles matching purely by chance; various modelling approaches have been proposed for estimating the haplotype frequencies and for adjusting drug efficacy estimates and novel genotyping approach has been recently suggested [10, 52-56]. For comparative studies, the results are confounded by the elimination half-lives of the drugs and the transmission setting in which the study is conducted. Advances in statistical methodologies and the availability of the methods in standard software programs have ensured that some of the issues raised in this review can now be easily addressed (Table 4). However, the true extent of the problem associated with these limitations is likely to have been overlooked, especially when individual trials are small and failures are few. It is important to define the extent of this bias, as derived efficacy estimates form the basis for driving policy decisions. The remit of the WorldWide Antimalarial Resistance Network Methods Study Group is well placed to answer some of these issues that will facilitate better methodologies and practices [44].

\section{Additional file}

Additional file 1. Additional tutorial, formulae and results.

\section{Authors' contributions}

PD, JAS, PJG, RNP and KS conceived the idea and wrote the first draft of the manuscript. All authors read and approved the final manuscript.

\section{Author details}

${ }^{1}$ WorldWide Antimalarial Resistance Network (WWARN), Oxford, UK. ${ }^{2}$ Centre for Tropical Medicine and Global Health, Nuffield Department of Clinical Medicine, University of Oxford, Old Road Campus, Roosevelt Drive, Oxford OX3 7FZ, UK. ${ }^{3}$ Centre for Epidemiology and Biostatistics, Melbourne School of Population and Global Health, The University of Melbourne, Melbourne, Australia. ${ }^{4}$ Department of Medicine, University of California, San Francisco, CA, USA. ${ }^{5}$ Global and Tropical Health Division, Menzies School of Health Research and Charles Darwin University, Darwin, Australia.

\section{Acknowledgements}

We thank Makoto Saito for thoroughly reviewing the manuscript and for several helpful discussions. We thank Christophe Combescure for help with the R package MetaSurv.

\section{Competing interests}

The authors declare that they have no competing interests.

\section{Funding}

PD is funded by Tropical Network Fund, Nuffield Department of Clinical Medicine, University of Oxford. The WorldWide Antimalarial Resistance Network (PD, KS, RNP, and PJG) is funded by a Bill and Melinda Gates Foundation grant and the ExxonMobil Foundation. JAS is an Australian National Health and Medical Research Council Senior Research Fellow (1104975). RNP is a Wellcome Trust Senior Fellow in Clinical Science (200909). The funders did not participate in the study development, the writing of the paper, decision to publish, or preparation of the manuscript.

\section{Publisher's Note}

Springer Nature remains neutral with regard to jurisdictional claims in published maps and institutional affiliations.

Received: 17 March 2017 Accepted: 19 October 2017

Published online: 26 October 2017 


\section{References}

1. White NJ. The assessment of antimalarial drug efficacy. Trends Parasitol. 2002;18:458-64.

2. White NJ, Pongtavornpinyo W, Maude RJ, Saralamba S, Aguas R, Stepniewska K, et al. Hyperparasitaemia and low dosing are an important source of anti-malarial drug resistance. Malar J. 2009;8:253.

3. WHO. Methods for surveillance of antimalarial drug efficacy. Geneva: World Health Organization; 2009.

4. WHO Guidelines for the treatment of malaria. 3rd ed. Geneva: World Health Organization; 2015.

5. Snounou G, Beck HP. The use of PCR genotyping in the assessment of recrudescence or reinfection after antimalarial drug treatment. Parasitol Today. 1998;14:462-7.

6. Slater M, Kiggundu M, Dokomajilar C, Kamya MR, Bakyaita N, Talisuna A, et al. Distinguishing recrudescences from new infections in antimalarial clinical trials: major impact of interpretation of genotyping results on estimates of drug efficacy. Am J Trop Med Hyg. 2005;73:256-62.

7. Cattamanchi A, Kyabayinze D, Hubbard A, Rosenthal PJ, Dorsey G. Distinguishing recrudescence from reinfection in a longitudinal antimalaria drug efficacy study: comparison of results based on genotyping of MSP1, MSP-2, and GLURP. Am J Trop Med Hyg. 2003;68:133-9.

8. Greenhouse B, Dokomajilar C, Hubbard A, Rosenthal PJ, Dorsey G. Impact of transmission intensity on the accuracy of genotyping to distinguish recrudescence from new infection in antimalarial clinical trials. Antimicrob Agents Chemother. 2007;51:3096-103.

9. Taylor AR, Flegg JA, Nsobya SL, Yeka A, Kamya MR, Rosenthal PJ, et al. Estimation of malaria haplotype and genotype frequencies: a statistical approach to overcome the challenge associated with multiclonal infections. Malar J. 2014:13:102

10. Messerli C, Hofmann NE, Beck H-P, Felger I. Critical evaluation of molecular monitoring in malaria drug efficacy trials: pitfalls of length polymorphic markers. Antimicrob Agents Chemother. 2016;61:01500-16.

11. Stepniewska K, Taylor WRJ, Mayxay M, Price R, Smithuis F, Guthmann J-P, et al. In vivo assessment of drug efficacy against Plasmodium falciparum malaria: duration of follow-up. Antimicrob Agents Chemother. 2004:48:4271-80

12. Stepniewska K, White NJ. Some considerations in the design and interpretation of antimalarial drug trials in uncomplicated falciparum malaria. Malar J. 2006;5:127.

13. Verret WJ, Dorsey G, Nosten F, Price RN. The effect of varying analytical methods on estimates of anti-malarial clinical efficacy. Malar J. 2009;8:77

14. Guthmann J-P, Pinoges L, Checchi F, Cousens S, Balkan S, van Herp M, et al. Methodological issues in the assessment of antimalarial drug treatment: analysis of 13 studies in eight African countries from 2001 to 2004 Antimicrob Agents Chemother. 2006;50:3734-9.

15. Covell G, Coatney GR, Field JW SJ. Chemotherapy of malaria. Monogr Ser World Health Organ. 1955

16. WHO. Resistance of malaria parasite to drugs. Geneva: World Health Organization; 1965

17. WHO. Assessment and monitoring of antimalarial drug efficacy for the treatment of uncomplicated falciparum malaria. Geneva: World Health Organization; 2003

18. WHO. Assessment of therapeutic efficacy of antimalarial drugs for uncomplicated falciparum malaria in areas with intense transmission. Geneva: World Health Organization; 1996.

19. Kaplan EL, Meier P. Nonparametric estimation from incomplete observations. J Am Stat Assoc. 1958:53:457-81.

20. Price RN, Nosten F, Luxemburger EK, Kham A, Brockman A, Chongsuphajaisiddhi $T$, et al. Artesunate versus artemether in combination with mefloquine for the treatment of multidrug-resistant falciparum malaria. Trans R Soc Trop Med Hyg. 1995;89:523.

21. WHO. Monitoring antimalarial drug resistance. Geneva: World Health Organization; 2001

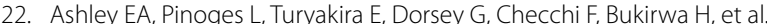
Different methodological approaches to the assessment of in vivo efficacy of three artemisinin-based combination antimalarial treatments for the treatment of uncomplicated falciparum malaria in African children. Malar J. 2008:7:154

23. Price RN, Dorsey G, Ashley EA, Barnes KI, Baird JK, D'Alessandro U, et al. World antimalarial resistance network I: clinical efficacy of antimalaria drugs. Malar J. 2007;6:119.
24. Gooley TA, Leisenring W, Crowley J, Storer BE. Estimation of failure probabilities in the presence of competing risks: new representations of old estimators. Stat Med. 1999:18:695-706.

25. Yeka A, Banek K, Bakyaita N, Staedke SG, Kamya MR, Talisuna A, et al. Artemisinin versus nonartemisinin combination therapy for uncomplicated malaria: randomized clinical trials from four sites in Uganda. PLoS Med. 2005;2:654-62

26. WorldWide Antimalarial Resistance Network. Data management and statistical analysis plan version 1.2. 2012. http://www.wwarn.org/sites/ default/files/ClinicalDMSAP.pdf. Accessed 23 Oct 2017.

27. Coviello V , Boggess M. Cumulative incidence estimation in the presence of competing risks. STATA J. 2004;4:103-12.

28. Pintilie M. Competing risks: a practical perspective. Chichester: Wiley; 2006.

29. Kalbfleisch JD, Prentice RL. Competing risks and multistate models. Stat Anal. Fail. Time Data. 2nd ed. New York: Wiley; 2002. p. 247-77.

30. Scrucca L, Santucci A, Aversa F. Competing risk analysis using R: an easy guide for clinicians. Bone Marrow Transplant. 2007:40:381-7.

31. Giolo SR. Turnbull's nonparametric estimator for interval-censored data Dep. Stat. Fed. Univ. Paraná. 2004. p. 1-10. http://www.est.ufpr.br/rt/ suely04a.pdf. Accessed 9 Dec 2016.

32. Turnbull BW. The empirical distribution function with arbitrarily grouped, censored and truncated data point in time is to be incorporated. J R Stat Soc. 1975;38:290-5.

33. Lindsey JC, Ryan LM. Tutorial in biostatistics. Methods for interval-censored data. Stat Med. 1998;17:219-38.

34. Gomez G, Calle ML, Oller R, Langohr K. Tutorial on methods for intervalcensored data and their implementation in R. Stat Model. 2009;9:259-97.

35. Singh RS, Totawattage DP. The statistical analysis of interval-censored failure time data with applications. Open J Stat. 2013;2013:155-66.

36. DerSimonian R, Laird N. Meta-analysis in clinical trials. Control Clin Trials. 1986;7:177-88

37. Higgins JP, Green S. Cochrane handbook for systematic reviews of interventions version 5.1.0. Cochrane Collab. 2008. http://community. cochrane.org/sites/default/files/uploads/Graph_recommendations9.pdf. Accessed 6 Dec 2015

38. Srinivasan C, Zhou M. A note on pooling Kaplan-Meier estimates. Biometrics. 1993:49:861-4

39. Rubin DB. An alternative to pooling Kaplan-Meier curves in time-toevent meta-analysis. Int J Biostat. 2011;7:1-26.

40. Combescure C, Foucher $Y$, Jackson D. Meta-analysis of single-arm survival studies: a distribution-free approach for estimating summary survival curves with random effects. Stat Med. 2014;33:2521-37.

41. Foucher Y, Combescure C, Jackson D. R Package MetaSurv. 2014. http:// www.divat.fr/en/softwares/metasurv. Accessed 11 Jan 2017.

42. Klein JP, Logan B, Harhoff M, Andersen PK. Analyzing survival curves at a fixed point in time. Stat Med. 2007;26:4505-19.

43. Arinaitwe E, Sandison TG, Wanzira H, Kakuru A, Homsy J, Kalamya J, et al. Artemether-lumefantrine versus dihydroartemisinin-piperaquine for falciparum malaria: a longitudinal, randomized trial in young Ugandan children. Clin Infect Dis. 2009:49:1629-37.

44. WorldWide Antimalarial Resistance Network. The WWARN Clinical Trials Methodology Study Group for P. falciparum. http://www.wwarn. org. 2016. http://www.wwarn.org/working-together/study-groups/ clinical-trials-methodology-study-group-p-falciparum. Accessed 24 Feb 2016.

45. Gray RJ. A class of K-sample tests for comparing the cumulative incidence of a competing risk. Ann Stat. 1988;16:1141-54.

46. Pintilie M. Analysing and interpreting competing risk data. Stat Med. 2007:26:1360-7.

47. Tai B-C, Wee J, Machin D. Analysis and design of randomised clinical trials involving competing risks endpoints. Trials. 2011:12:127.

48. Borrmann S, Peto T, Snow RW, Gutteridge W, White NJ. Revisiting the design of phase III clinical trials of antimalarial drugs for uncomplicated Plasmodium falciparum malaria. PLoS Med. 2008;5:e227.

49. FDA. Malaria : developing drug and nonvaccine biological products for treatment and prophylaxis. 2007. https://www.fda.gov/OHRMS/ DOCKETS/98fr/07d-0212-gdl0001.pdf. Accessed 23 Oct 2017.

50. Com-Nougue C, Rodary C, Patte C. How to establish equivalence when data are censored: a randomized trial of treatments for B non-Hodgkin lymphoma. Stat Med. 1993;12:1353-64. 
51. da Silva GT, Logan BR, Klein JP. Methods for equivalence and noninferiority testing. Biol Blood Marrow Transplant. 2009;15:120-7.

52. Gatton ML, Cheng Q. Can estimates of antimalarial efficacy from field studies be improved? Trends Parasitol. 2008;24:68-73.

53. Porter KA, Burch CL, Poole C, Juliano JJ, Cole SR, Meshnick SR. Uncertain outcomes: adjusting for misclassification in antimalarial efficacy studies. Epidemiol Infect. 2011;139:544-51.

54. Jaki T, Parry A, Winter K, Hastings I. Analysing malaria drug trials on a per-individual or per-clone basis: a comparison of methods. Stat Med. 2013;32:3020-38
55. Plucinski MM, Morton L, Bushman M, Dimbu PR, Udhayakumar V. Robust algorithm for systematic classification of malaria late treatment failures as recrudescence or reinfection using microsatellite genotyping. Antimicrob Agents Chemother. 2015;59:6096-100.

56. Ken-Dror G, Hastings IM. Markov chain Monte Carlo and expectation maximization approaches for estimation of haplotype frequencies for multiply infected human blood samples. Malar J. 2016;15:430.

57. Fay MP. Weighted logrank tests and NPMLE for interval censored data. R package "interval." CRAN Repos. 2015.

\section{Submit your next manuscript to BioMed Central and we will help you at every step:}

- We accept pre-submission inquiries

- Our selector tool helps you to find the most relevant journal

- We provide round the clock customer support

- Convenient online submission

- Thorough peer review

- Inclusion in PubMed and all major indexing services

- Maximum visibility for your research

Submit your manuscript at www.biomedcentral com/submit 